\title{
The Effect of Dynamic Interfragmentary Movement on the Mechanical Performance of External Fixation Devices for Open Tibial Fractures
}

\author{
Thongchai Leetha, Veena Phunpeng and Supakit Rooppakhun* \\ School of Mechanical Engineering, Institute of Engineering, Suranaree University, \\ 111 University Avenue, Muang Nakhon Ratchasima 30000, Thailand \\ *Corresponding Author: supakit@sut.ac.th
}

\begin{abstract}
Interfragmentary movement (IFM) in bone formations during tibial shaft fracture stabilization is known to influence the outcome of fracture healing, as well as the performance of external fixation devices. Theoretically, the construction stiffness of a fixation device, including pin insertion configuration, is associated with tibial fracture stability. The objective of this study is to investigate the effect of interfragmentary movement during tibial fracture healing time using finite element analysis, with particular regard for the construction stiffness of the external fixation device. The effect of the distance between pin insertion and the pin length on the mechanical performance of the external fixation device was also performed, with reference to the ASTM F1541 standard. The mechanical stiffness ratio between the fracture site and skeleton device during each healing phase was calculated. According to the numerical results, the axial stiffness of the device displayed values in the range of 130 $950 \mathrm{~N} / \mathrm{mm}$. The effect of the pin length on the construction stiffness was found to be higher than the effect of the distance between pin insertions. It is implied that the load sharing between the stabilization occurred in fixation device and callus during first seven weeks.
\end{abstract}

Keywords: External fixation, Inter fragmentary movement, Open tibial fracture, Mechanical performance

\section{Introduction}

Open factures of the tibia caused by high-energy trauma are the most common type of long-bone in hurry. Generally, the objectives of tibial shaft fracture management are the prevention of infection, achievement of bony union, and the restoration of function. An external fixation device is clinically recommended for definitive or provisional stabilization of the fracture until soft tissue recovery and complete bone healing have occurred. Normally, external fixators are designed to provide sufficient interfragmentary motion (called the "static phase") to stimulate secondary bone healing by callus formation (called the "dynamics phase").

According to the process of fracture healing, the natural bone regeneration consists of the inflammation, reparative and remodeling phases, as shown in Fig 1. The inflammation phase is the earliest stage of bone fracture healing, and ends when the bone begins its formation, which will take about 34 days or 10 percent of the total time. During this phase, a hematoma is formed in the reparative phase a proliferation of cartilage cells from the inner cell membrane skeleton occurs, and the hematoma is converted to fibrous tissue and cartilage. A hard callus is formed which is capable of generating the texture of the bone formation. This phase takes about 3-4 weeks or 40 percent of the time. Finally, the remodeling phase occurs. During this period, there will be a decrease and increase new bone structure inside the cell

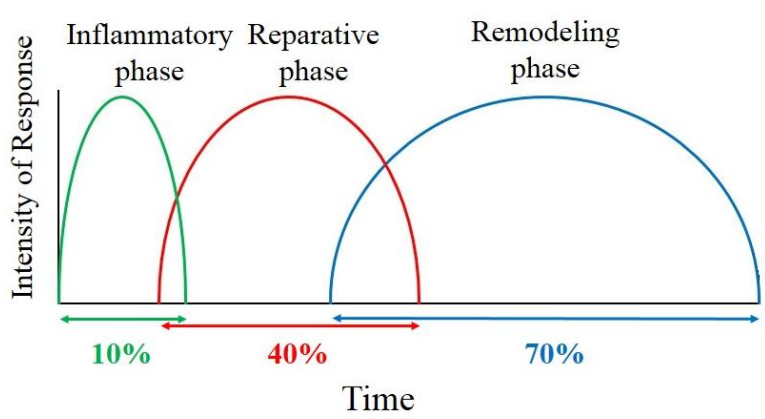

Fig 1. The process of fracture healing. 
through a temporary soft bone formation and breakdown of bone cells. This period can take anything from several months to years. However, even after the repair has been completed successfully the effectiveness of the repaired bone is often inferior to the bone before. Stimulation of the bone during healing has been suggested to improve the potential bone fracture mineralization and biomechanical properties, with the stimulus being movement in the area of the bone fracture, which is known as interfragmentary motion $(\mathrm{IFM})^{(1,2)}$.

Commercially there are three types of external fixation device in common use: the unilateral external fixator; the Illizarov external fixator; and the hybrid external fixator. Each of these has 3 major components: the schanz screw (or pin), a set of clamps, and a body (in the form of rods and/or rings), as illustrated in Fig. 2. It can also allow for the implementation of an additional mechanism, called a dynamization external fixation. The function of this device is as a mobile device that transmits axial movement to the fracture, using the stiffness of the fixator to influence the transfer motion.

The unilateral external fixation device consists of a rod positioned on one side of the fractured bone, and is simple and quick to install. The distance between the pins can easily be aligned and adjusted to match the characteristics of the fracture. It can act to stabilize the fracture as much as

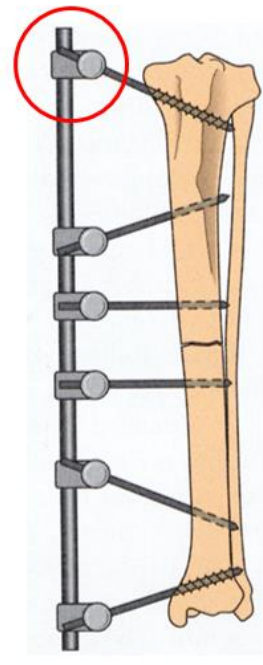

(a)

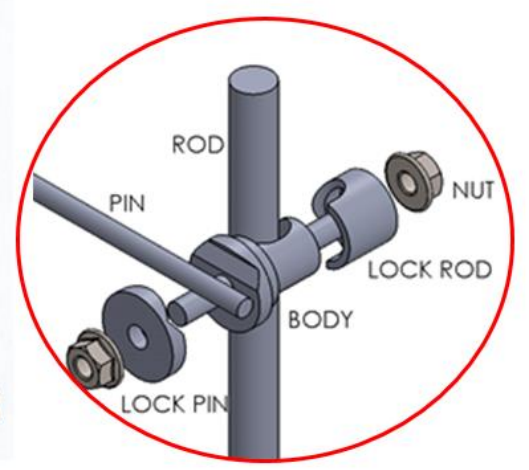

(b)
Fig 2. (a). Unilateral External fixation device.

(b). Detail components of unilateral External fixation device. possible, but at the cost of the patient being unable to fully put weight on the leg with the device. A properly applied fixator may improve the healing process, while one improperly applied might delay the healing process ${ }^{(4)}$. The use of an external fixator can also allow for the stimulation of fracture healing by application of IFM in the axial direction of the fractured bone, using a dynamization external fixation. This can benefit the quality of the healed bone, for example by resulting in an increase tensile strength, bone mineral density, and/or bending stiffness of the newly formed bone ${ }^{(5,6)}$ when compared to a fracture that has been rigidly fixed.

This study aims to investigate the effect of dynamic interfragmentary movement on the mechanical performance of an external fixation device for open tibial fracture by creating a model for a unilateral external fixator, so that the effects of interfragmentary movement and pin insertion can be investigated. This can allow for the optimum performance in fracture healing to be determined.

\section{Materials and Methods}

In this study, the external fixation device was created using computer-aided design (CAD) software (in this case SolidWorks 2015). The device consists of pins 5.5-mm in diameter, clamps (consisting of a lock rod, body, lock pin and nuts), and a rod 11-mm in diameter, as shown in Fig 2. To simplify the modelling, acrylic rods $30-\mathrm{mm}$ in diameter were used as the tibia bone models, which are herein referred to as the polyacetal (POM).

The model of the external fixation device was analyzed to take into consideration the assembly requirement of the ASTM (American Society for Testing and Materials) technical standard F1541-standard specification and test methods for skeletal fixation devices ${ }^{(7,8)}$. The details of the assembly are represented in Fig 3 and Table 1.

For all the components of the system it was assumed that the behavior of the material was homogeneous, linear, elastic and isotropic ${ }^{(9)}$, and could be defined using the modulus of elasticity E and Poisson's coefficient. The values used for each of the components are given in Table 2.

In the modelling, a weight of $7 \mathrm{Kg}$ person was applied to the POM, which corresponds to 10 percent of the $70 \mathrm{Kg}$ weight of an average person ${ }^{(11-14)}$.

The callus stiffness $(\mathrm{Kc})$ is related to the interfragmentary movement (IMF) and axial reaction force, and was assumed from estimating the walking conditions which trigger the fracture healing outcome ${ }^{(15)}$ 

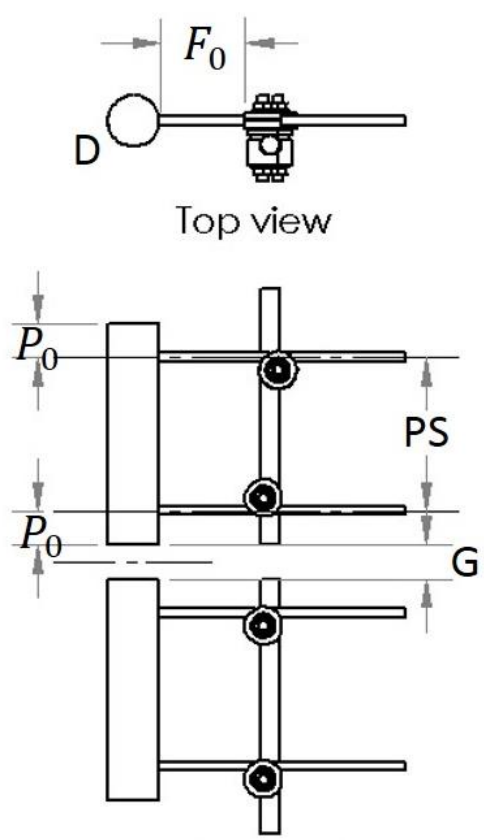

Frontal view

Fig 3. External Fixator assembly F1541-Standard Specification.

Table 1. Assembly parameters (F1541).

\begin{tabular}{|c|c|}
\hline PS & $90 \mathrm{~mm}$ \\
\hline$F_{0}$ & $50 \mathrm{~mm}$ \\
\hline$P_{0}$ & $20 \mathrm{~mm}$ \\
\hline $\mathrm{G}$ & $20 \mathrm{~mm}$ \\
\hline $\mathrm{D}$ & $30 \mathrm{~mm}$ \\
\hline
\end{tabular}

Table 2. Properties of the materials ${ }^{(9)}$.

\begin{tabular}{|c|c|c|c|}
\hline Description & Material & E( GPa ) & v \\
\hline Pin & $\begin{array}{c}\text { Stainless SUS } \\
\text { 316LVM }\end{array}$ & 200 & 0.33 \\
\hline Clamp, Nut & Stainless SUS 304 & 190 & 0.29 \\
\hline Rod & Carbon fiber & 135 & 0.30 \\
\hline POM & Polyoxymethylene & 2.5 & 0.30 \\
\hline
\end{tabular}

Finite Element (FE) analysis was performed on the assembly using a commercial FE software package (ANSYS Workbench). In the analysis, 2-mm tetrahedral elements were used to generate the FE model. For the contact conditions, all contact areas were assumed frictionless ${ }^{(16)}$. The contacts between two adjacent objects were restricted to having no relative movement, with the exception of the contact between the pin and the acrylic POM which allowed relative displacement ${ }^{(9)}$.

\section{Results}

\subsection{Effect of interfragmentary movement}

Using the external fixation device assembly configuration as defined in ASTM F1541, the axial stiffness of the device from the finite element simulation was found to be $205.28 \mathrm{~N} / \mathrm{mm}$. This is shown in Table 3, along with the values obtained in previous studies. Fig. 4 shows the relationship between the stiffness ratio [defined as the Callus stiffness, $(\mathrm{Kc})$ per Device stiffness, $(\mathrm{Kd})]$ and healing time. The figure shows an increase in the stiffness ratio during the healing process. Note that the stiffness ratio in first seven weeks is relatively low, but shows a significant increase after the seventh week of the healing process. The cause of this is due to the soft tissue bone have less strength and more the

Table 3. Construction stiffness

\begin{tabular}{|c|c|}
\hline External Fixation System & $\begin{array}{c}\text { Axial Stiffness } \\
(\mathrm{N} / \mathrm{mm})\end{array}$ \\
\hline${\text { Yang et } \mathrm{al}^{(17)}}^{(126.00}$ \\
\hline${\text { Mesic et } \mathrm{al}^{(18)}}$ & 143.00 \\
\hline Tananan et $\mathrm{al}^{(9)}$ & 166.50 \\
\hline Present Study $^{(19)}$ & 205.28 \\
\hline${\text { Koo et } \mathrm{l}^{(19)}}$ & 246.00 \\
\hline Marcelo et $\mathrm{al}^{(7)}$ & 307.60 \\
\hline
\end{tabular}

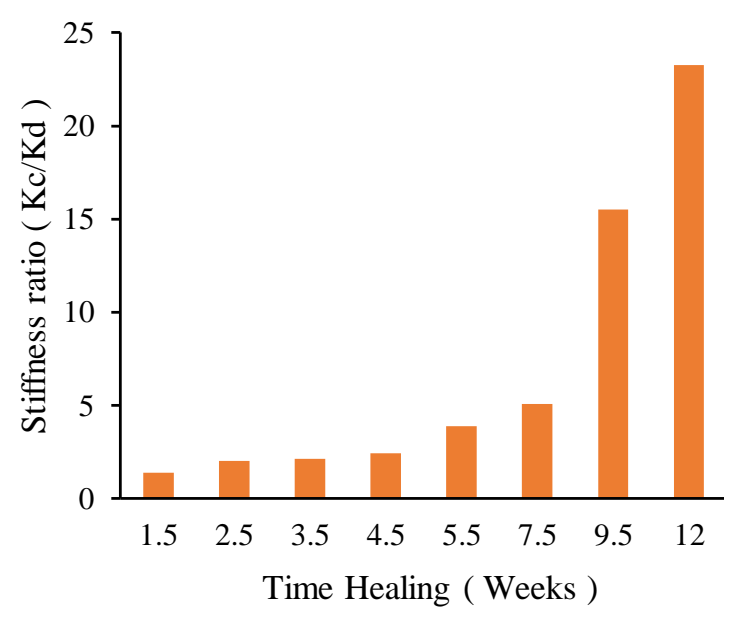

Fig. 4. Relative stiffness ratio $(\mathrm{Kc} / \mathrm{Kd})$ at different times during the healing process. $\mathrm{Kc}$ is the stiffness of the callous, obtained from experimental data ${ }^{(15)}$ and $\mathrm{Kd}$ is the stiffness of the external fixation device calculated from the present FE model. 
movement of bone fracture during the first seven weeks of healing. The stiffness ratio will influence the displacement and force on the external fixation device, and hence the displacement and force on the bone fracture according to a relationship given by the following equation:

$$
\delta_{d}=k \frac{F_{d} \cdot \delta_{c}}{F_{c}}
$$

Where $\delta_{d}$ is a device displacement, $\delta_{c}$ is the displacement of bone fracture, $F_{d}$ is the axial load with the device, $F_{c}$ is the axial load with bone fracture, $k$ is the stiffness ratio.

\subsection{Effects of $F_{0}$ and $P S$ parameter of pin insertion.}

(a) Strength.

The stress distribution under axial loading conditions is presented in Fig 5. The magnitude of maximum stress values in the case of axial loading presented in table 4 and 5.

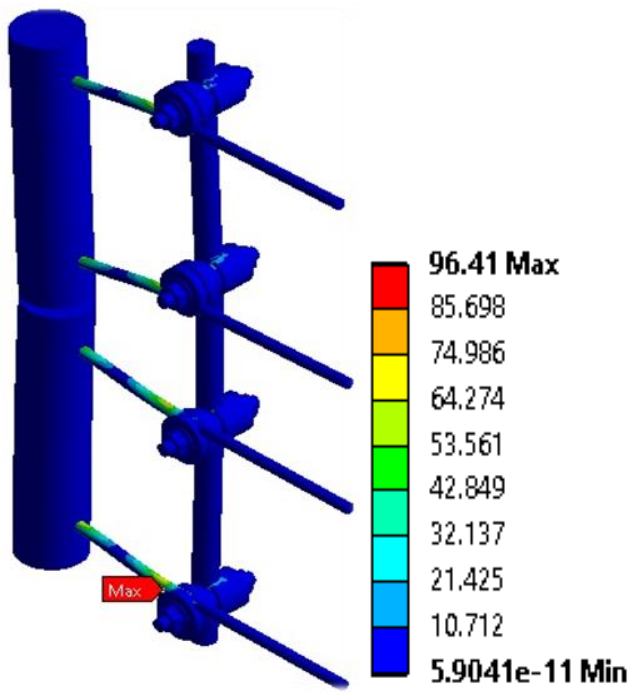

(a)

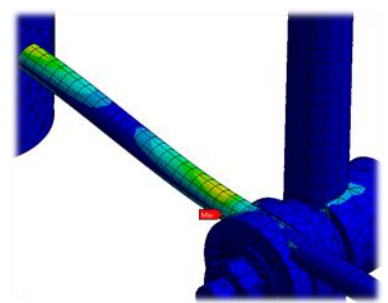

(b)

Fig. 5 Show the 3D FE model of the analyzed Unilateral External fixation device after the action of axial load 70 N. Maximum stress of structure of vary pin insertion occur on pin of structure are represent in Table 4 and 5 .
Table 4. Influenced of pin length.

\begin{tabular}{|c|c|}
\hline $\begin{array}{c}F_{0} \\
(\mathrm{~mm})\end{array}$ & $\begin{array}{c}\text { Maximum stress } \\
(\mathrm{MPa})\end{array}$ \\
\hline 30 & 46.53 \\
\hline 40 & 77.00 \\
\hline 50 & 96.41 \\
\hline 60 & 97.81 \\
\hline 70 & 110.43 \\
\hline
\end{tabular}

Table 5. Influenced of distance between pin insertions.

\begin{tabular}{|c|c|}
\hline $\begin{array}{c}P S \\
(\mathrm{~mm})\end{array}$ & $\begin{array}{c}\text { Maximum stress } \\
(\mathrm{MPa})\end{array}$ \\
\hline 70 & 95.52 \\
\hline 80 & 95.79 \\
\hline 90 & 96.41 \\
\hline 100 & 97.02 \\
\hline 110 & 97.49 \\
\hline
\end{tabular}

(b) Stiffness.

Fig. 6 shows the relationship of the horizontal displacement $\left(F_{0}\right)$ of the external fixation device to the stiffness of the device $\left(K_{d}\right)$. Included are 30, 40, 50, 60, and $70 \mathrm{~mm}$ displacements. The results from the simulation of the assembly at increase displacement $\left(F_{0}\right)$ result in a reduced device stiffness $\left(K_{d}\right)$. Conversely, reducing the displacement $\left(F_{0}\right)$ increases the device stiffness $\left(K_{d}\right)$.

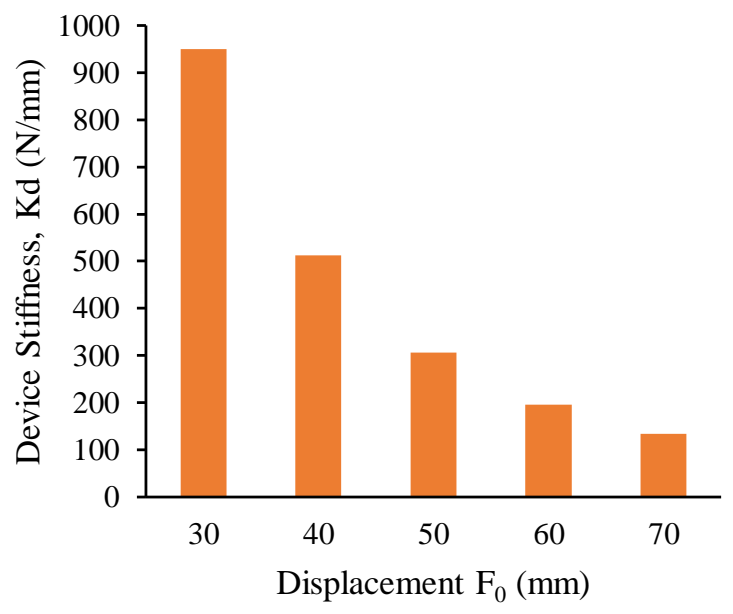

Fig. 6. Relative of Device stiffness (Kd is the stiffness of device form the FE model.) with displacement assembly parameters $F_{0}$ of F1541 Standard Specification. 


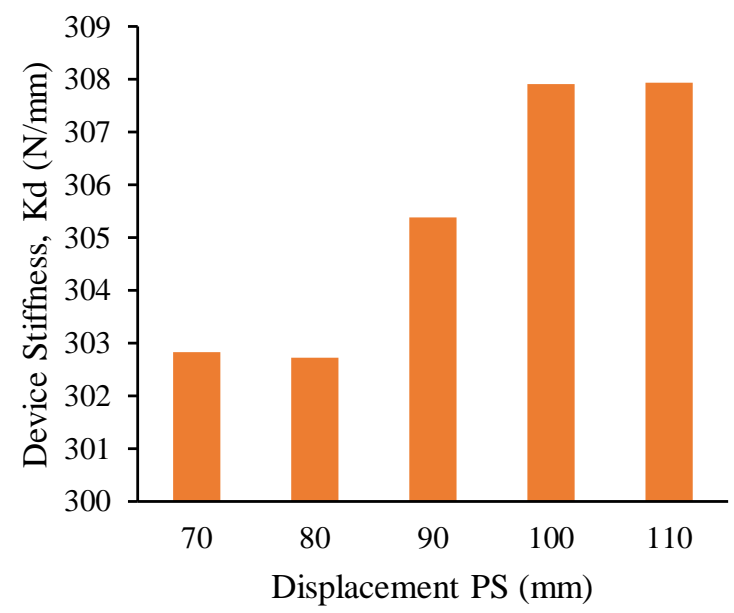

Fig. 7 Relative of Device stiffness (Kd is the stiffness of device form the FE model.) with displacement assembly parameters PS of F1541 Standard Specification.

Fig 7 shows the relationship between the vertical displacement (PS) of the external fixation device pins and the stiffness of the device $\left(K_{d}\right)$. Included are $70,80,90,100$, and $110 \mathrm{~mm}$ displacements. The results from the simulation show that an increased displacement (PS) will result in an increase in the device stiffness $\left(K_{d}\right)$. Conversely, the results from the simulation of the assembly at reduced displacement (PS) suggest a reduction in the device stiffness $\left(K_{d}\right)$.

The simulation results for adjusting parameters $\mathrm{F}_{0}$ and PS compliance with behavior stiffness of external fixation device suggest that the $F_{0}$ values affect the behavior of stiffness more than the varying the PS value by a factor of $53.65 \%$.

The axial stiffness of the device under study had a stiffness value that is more than device by Yang et al ${ }^{(17)}$. Mesic et al ${ }^{(18)}$, and Tananan et al ${ }^{(15)}$ by approximately $62.92 \%, 43.55 \%$, and $23.35 \%$ respectively, and lower than device by Koo et al ${ }^{(19)}$, and Marcelo et al (7) by approximately $16.55 \%$ and $33.26 \%$ respectively.

\section{Discussion}

For construction stiffness, the results from the present study are compared to the other results in the literature in Table 3. There are a wide range of values for the stiffness in the literature. This contrast occurs from the clamp design of the external fixation device, and the stiffness can be varied by varying the displacement of assembly device.

The results show that the stiffness ratio increases with time, the while device makes the bone fracture movement decrease with time. It is evident that a greater likelihood of the displacement of the rod happening results in an decrease in the healing time. However, in the stimulation of bone fracture movement, the movement was only active for the first five weeks or Reparative phase ${ }^{(20)}$, not the full 12 weeks of healing time.

The outcome of varying the displacement $\left(F_{0}, P S\right)$ of the assembly suggests that the healing time could be greatly reduced by modification of the fixator design to increase the fixation stiffness. When using external fixation devices, this could be achieved by decreasing the free bending length of the pins, using a stiff fixation body and a stiff connection between the pins and the body ${ }^{(16)}$. However, in this study we investigated external fixation devices by decreasing the length of the pins, which also resulted in an increase in the stiffness of the external fixation device, as shown in Fig. 4 and Fig. 5. In this study, the assembly used was defined by ASTM F1541. However in treatment, the positions of fixators are usually defined by surgeons based on his/her own experience. Although from a clinical point of view there are several factors that can determine the pin and side bar positions, an optimized model could contribute to the best clinical decision. Generally, for treatment of an open type tibial fracture an external fixation device will use a rigid fixator. For better results, this device should be working in both rigid and flexible conditions. Flexible devices could be moved into the canal during the healing time. External fixation devices should be able to control the reduction of bone fracture axial movement as the healing time progresses and/or allow for inverse dynamization ${ }^{(20)}$.

\section{Conclusions}

The results demonstrate that the greater displacement PS relates to greater device stiffness, and also that a lower displacement $\mathrm{F}_{0}$ provides a greater device stiffness. This will affect the bone fracture movement during healing, leading to improved mechanical properties of the bone after the healing process has finished.

\section{Acknowledgment}

The authors would like to acknowledge Suranaree University of Technology for funding and facility support during these research activities, and I would like to deeply thank Dr. James Varley for his helpful comments on the work. 


\section{References}

(1) Marsell R, and Einhom $\mathrm{T}$ : “The biology of fracture healing", Injury, Vol.42, No.6, pp.551-555, 2011

(2) J.A. Alierta, M.A. Perez, and J.M. Garcia-Aznar : “An interfrace finite element model can be used to predict healing outcome of bone fracture", Journal of Mechanical Behavior og Biomedical Materials, Vol.29, pp.328-338, 2014

(3) Clase LE, Recknagel S, and Ignatius A : "Fracture healing under healthy and inflammatory conditions", Nat Rev Rheumatol, Vol.8, No.3, pp.133-143, 2012

(4) Luis M. Roseiro, M.Augusta Neto, Ana Amaro, Rogerio P. Leal, and Miguel C. Samarra : "Computer Methods and Programs in Biomedicine", Vol.13, pp.360-370, 2014

(5) L E Claes, H-J Wilke, P Augat, S Rubenacker, and K J Margevicius : "Effect of dynamization on gap healing of diaphyseal fractures under external fixation", Clinical Biomechanics, Vol.10, No.5, pp.227-234, 1995

(6) S. Wolf, A. Janousek, J. Pfeil, W. Veith, E. Haas, G. Duda, and L. Claes : "The effects of external mechanical stimulation on the healing of diaphyseal osteomies fixed by flexible external fixation", Clinical Biomechanics, Vol.13, pp.359-364, 1998

(7) Marcelo Back Sternick, Darlan Dallacosta, Daniela Aguida Bento, and Marcelo Lemos do Reis : "Reationship between rigiditt of external fixator and number of pin : computer analysis using finite elements", Rev Bras Ortop, Vol.47, No.5, pp.646-650, 2012

(8) ASTM Comitee F04 on Medical and Surgical Materials and Devices. Designation F 1541-2. Standard Speccification and Test Methods for External Skeletal Fixation Devices, 2002

(9) Thananan Sisuphan, Supakit Roopakhun, Yingyong Suksathien, and Nattapon Chantarapanich : "Mechanical Evaluation of the first Generation of Korat Static External Fixtation Device", IPCSIT, Vol.23, pp44-47, 2012

(10)Heintz S and Gutierrez-Farewik EM : "Static optimization of muscle forces during gait in comparison to EMG-to-force processing approcach”, Gait Posture, Vol.1, No.3, pp.247-250, 2007

(11) Anderson FC and Pandy MG : "Static and dynamic optimization solutions for gait are practically equivalent”, Journal Biomechanics, Vol.26, pp.279-288, 2007
(12) Kim S-H, Chang S-H, and Son D-s : "Finite element analysis of the effect of bending stiffness and contact condition of composite bone plates with simple rectangular cross-section on the bio-mechanical behavior of fractures long bong", Compos Part B, Vol.42, pp.1731-1738, 2001

(13) J.T-M. Cheung, M. Zhang, A.K.L. Leung, and Y.B. Fan : "Three-dimensional finite element analysis of the foot during standing-a material sensitivity study", Journal Biomechanics, Vol.38, pp.1045-1054, 2005

(14) A. Simkin : "Structural analysis of the human foot in standing posture", Tel Aviv : Tel Aviv University, 1982

(15) Tim Wehner, Lutz Claes, Frank Niemeyer, Daniel Nolte, and Ulrich Simon: "Influence of the fixation stability on the healing time - A numerical study of a patientspecific fracture healing process", Vol.25, pp.606-612, 2010

(16) Karunratanakul Kavin, Schrooten Jan, and Oosterwyck Hans-van : "Finite Element Modeling of a Unilateral Fixator for Bone Reconstruction : Importance of Contact Setting", Journal Medical Engineering and Physics, Vol.32, pp.461-467, 2010

(17) Yang lang, Nayagum selvadurai, and Saleh Michael : "Stiffness Characteristics and Interfragmentary Displacement with Different Hybrid External Fixator", Clinical Biomechanics, Vol.18, No.2, pp.166-172, 2003

(18) Mešić Elmedin, Avdić Vahid, Pervan Nedim, and Repčić Nedžad : "Finite Element Analysis and Experimental Testing of Stiffness of the Sarafix External Fixator", Procedia Engineering, Vol.100, pp.1589-1607, 2015

(19) Terry-Kwok-Keung Koo, Kim young, DB chooi, KG hua, Lim gino, Inoue nozomu, and Eys chao : "Stiffness Analysys of dynafix external fixator system", ASME Summer Bioengineering conference, 2003

(20) Elaine N. Marieb, and Katja Hoehn : "Human Anatomy \& Physiology", pearson, 2013

(21) D.R. Epari, T. Wehner, A. Ignatius, M.A. Schuetz, and L.E. Claes : "A case for optimising fracture healing through inverse dynamization", Medical Hypotheses, Vol.81, pp.225-227, 2013 